\title{
Use of cylindrical samples for ceramics strength measurements
}

\author{
Jean-Claude Glandus ${ }^{1}$, Joseph Absi ${ }^{1, a}$, Michel Saliceto ${ }^{2}$ and Philippe Fougerolle ${ }^{2}$ \\ 1 G.E.M.H., ENSCI, 47 avenue Albert Thomas, 87065 Limoges, France \\ 2 CEA DAM, Centre d'Études du Ripault, BP 16, 37260 Monts, France
}

Received 23 February 2004, Accepted 7 March 2006

\begin{abstract}
In spite of his great applied interest, the tensile strength of brittle materials, and chiefly that of ceramics, is difficult to measure directly by classical tensile tests. This results from the difficulties induced both by the samples machining and their grip on the testing machine. So, it is common to perform indirect determinations on the basis of strength values obtained in 3 or 4 points bending tests on prismatic samples. In the case of massive or hollow cylindrical samples, the diametral compression tests seem to be the best measurement technique, but these tests require refined experimental approach to lead to significant results. In order to characterise these care, two original experimental devices, one dedicated to massive cylinders, the other to hollow cylinders, have been built. The comparison of results thus obtained with those given by the classical bending tests permits to specify the validity and the limits of the techniques here developed.
\end{abstract}

Key words: Ceramics / tensite strength / diameteral compression / finite element method

Résumé - Utilisation d'éprouvettes cylindriques pour la mesure de la résistance à la rupture des céramiques. Bien qu'elle présente un intérêt appliqué primordial, la résistance à la rupture en traction des matériaux fragiles, et plus particulièrement celle des matériaux céramiques, est difficile à mesurer directement par des essais de traction classiques. Ceci résulte des difficultés liées à l'usinage des éprouvettes ainsi qu'à leur maintien dans un système d'amarrage. De ce fait, la pratique privilégie des déterminations indirectes à partir d'essais de flexion 3 ou 4 points sur des éprouvettes prismatiques. Dans le cas courant d'éprouvettes cylindriques pleines ou creuses, des essais de compression diamétrale semblent être la meilleure technique expérimentale, mais ces essais réclament une approche expérimentale raffinée pour conduire à des résultats significatifs. En vue de caractériser les précautions nécessaires, deux montages expérimentaux originaux, l'un destiné aux cylindres pleins, l'autre aux cylindres creux, ont été réalisés. La comparaison des résultats ainsi obtenus avec ceux donnés par les essais classiques de flexion permet de préciser la validité et les limites de cette approche.

Mots clés : Céramique / résistance à la rupture en traction / compression diamétrale / méthode des éléments finis

\section{Introduction}

Tensile strength of ceramics is a crucial mechanical data whose knowledge is essential, both from a theoretical view point (the fracture mechanics uses frequently this parameter) and from an applied one (mechanical calculation of pieces and components needs such data). Unfortunately, its fine experimental determination is always difficult because ceramic materials cannot be easily tested in the same way than metallic samples. On one hand, it is difficult and expensive to make shouldered samples and,

\footnotetext{
a Corresponding author: j_absi@ensci.fr
}

on the other hand, the full suppression of disturbing bending effects requires sophisticated experimental setups.

To get round these difficulties, the ceramics strength measurement is generally performed by means of 3 or 4 points bending tests. Such tests are easy to perform and the preparation of samples is limited to grinding and polishing operations. The distribution of normal stresses in a cross section is not uniform, but starting from the material Weibull's modulus it is possible to estimate the pure tensile $\sigma_{\mathrm{R}}$ value [1].

The bi-axial bending test [2] is well suited to test samples in shape of disks, which are easy to make by the classical shaping ceramic techniques. It presents the advantage to be apparently easy to carry out and to be not 


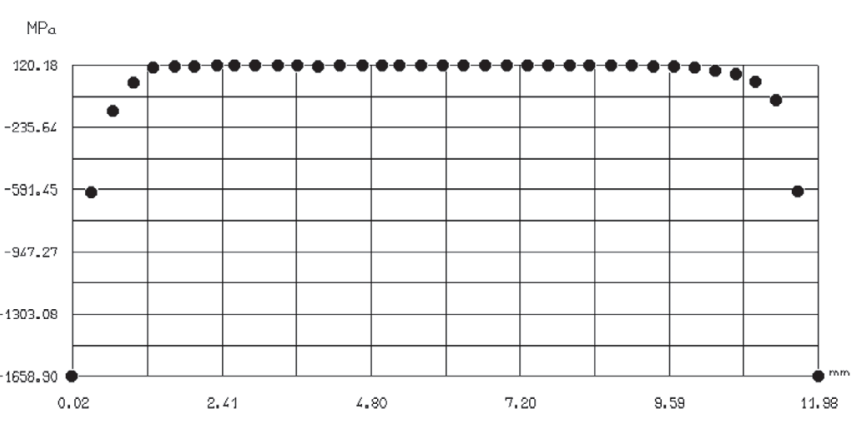

Fig. 1. $\sigma_{X X}$ stresses in the diametral vertical plane.

sensitive to edge effects. However, it leads to strength values difficult to compare with those given by the previous uni-axial tests and, moreover, only a weak part of the whole sample volume is subjected to tensile stresses.

Finally, the diametral compression of cylinders (which are also samples easy to obtain by usual ceramic technologies), is a rather few used testing method in spite of its ability to lead to the tensile strength of brittle materials. Its principle consists to apply two compressive loads along diametrically opposite generating lines of a cylindrical sample maintained between the plates of a testing machine.

The present paper is devoted to the use of this test in the case of massive and hollow cylinders. A special attention is given to the original solutions proposed to get round experimental problems resulting from the necessity to warrant a double linear contact between the sample and the loading plates. If such a contact is not insured, the diametral compression test becomes a crush corner one.

\section{Diametral compression of massive cylinders}

This test, also called "brazilian test" [3,4], is very used by geologists and civil engineers. It calls for high loads and its main difficulty arises from the necessity to use an experimental device both robust and sufficiently compliant to allow the auto alignment of the compressive loading plates along two opposite generating lines of the sample.

\subsection{Stress field}

Many works have been devoted to the survey of the stress field in a cross section of cylinders subjected to diametral compression $[5,6]$. They describe the $\sigma_{\mathrm{R}}$ variations along the vertical and horizontal diameters of a cross section and take into account the localised sample deformation in the vicinity of the zones of contact. They show that sufficiently far from these zones, the vertical diametral plane is loaded by quite constant normal tensile stresses $\sigma_{X X}$ and that, along the same direction, the compressive stresses $\sigma_{Y Y}$ are always very high as illustrated by Figures 1 and 2 (values obtained by F.E.M. simulation of the test).

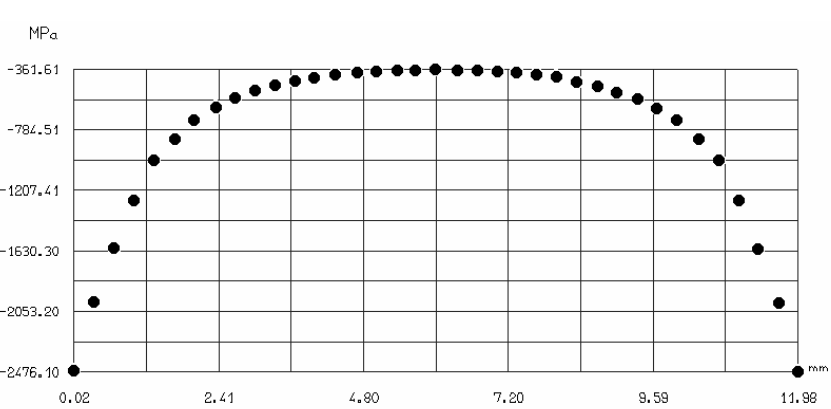

Fig. 2. $\sigma_{Y Y}$ stresses in the diametral vertical plane.

If the contact between the sample and the loading plates is perfectly linear, the cracking starts from the centre of the sample under tensile stresses whose magnitude is given by the relationship:

$$
\sigma_{r}=\sigma_{\theta}=\frac{2 P}{\pi D L}
$$

( $P$ is the fracture load, $D$ the sample diameter and $L$ its length).

\subsection{Experimental device and samples}

The original experimental device used (cf. Figs. 3 and 4 ) is constituted by a lower bearing plate and a upper mobile plate guided by 2 columns provided with linear ball bushings.

The upper plate is in contact with the cross head of the testing machine and transmits the load to the sample. The lower plate is provided with an hemispheric bearing area supporting the anvil on which the sample is put. To rest, this anvil is not in contact with the bearing area but is maintained in floating position, slightly over the hemispheric bearing, by means of compliant springs embedded in the lower plate. Thus, at the beginning of loading, a perfect linear contact can be achieved between two opposite generating lines of the sample and the loading plates. So, this previous auto alignment is maintained when the contact occurs between the anvil and the hemispheric bearing area.

Finally, two elastic lateral guides, which push on the two generating lines located in the horizontal diametral plane, maintain the sample in the axis of the device.

\subsection{Device validation}

\subsubsection{Experimental design}

Because many experimental parameters are able to affect the measured strength values, a reduced experimental design has been performed to characterise the respective influences of these parameters.

This experimental design, using Taguchi's method [7] without interaction (cf. Tab. 1), takes into account 7 parameters (considered as significant) to characterise the 

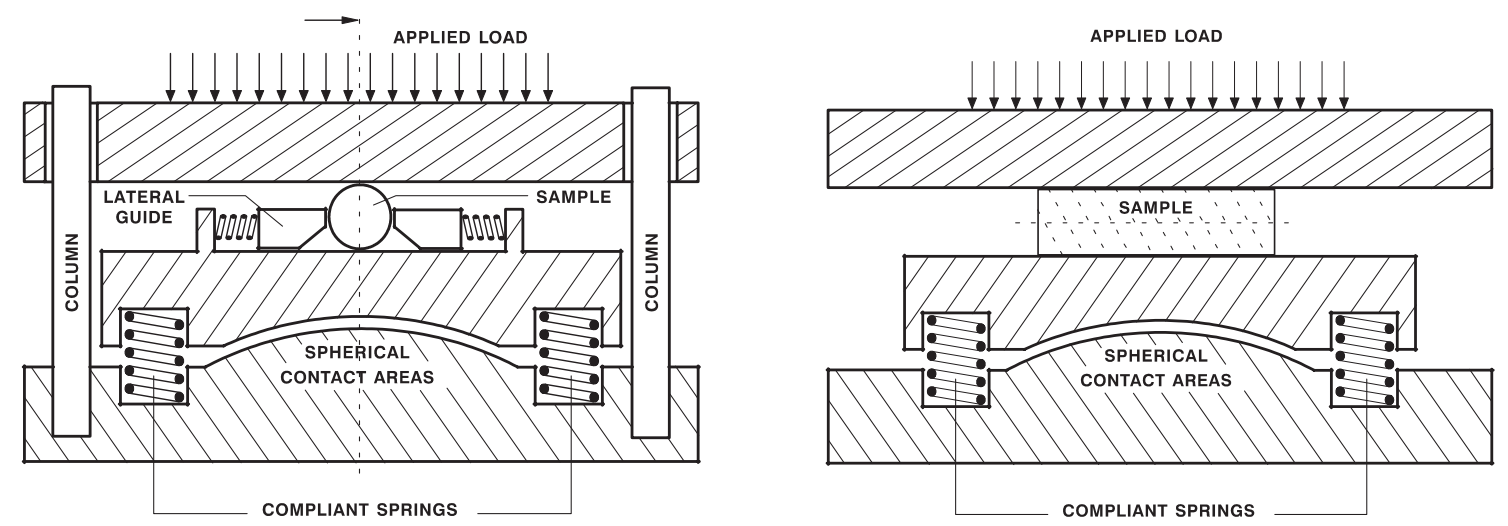

Fig. 3. Principle of the experimental device for diametral compression of massive cylinders.

Table 1. Taguchi's table for the experimental design.

\begin{tabular}{|c|c|c|c|c|c|c|c|}
\hline Parameters & $\mathbf{A}$ & $\mathbf{B}$ & $\mathbf{C}$ & $\mathbf{D}$ & $\mathbf{E}$ & $\mathbf{F}$ & $\mathbf{G}$ \\
\hline Case & $\begin{array}{c}\text { Loading } \\
\text { rate } \\
\left.\mathrm{mm}^{\mathrm{min}} \mathrm{m}^{-1}\right)\end{array}$ & $\begin{array}{c}\text { Half-flats } \\
(1 \mathrm{~mm})\end{array}$ & $\begin{array}{c}\text { Diameter } \\
(\mathrm{mm})\end{array}$ & $\begin{array}{c}\text { Brass } \\
\text { Pads }\end{array}$ & $\begin{array}{c}\text { Surfacic } \\
\text { defects }\end{array}$ & $\begin{array}{c}\text { Conicity } \\
(\mathrm{mm})\end{array}$ & $\begin{array}{c}\text { Lateral } \\
\text { guides }\end{array}$ \\
\hline 1 & 0.2 & No & 12 & No & No & 0 & Yes \\
\hline 2 & 0.2 & No & 12 & No & No & 0.2 & No \\
\hline 3 & 0.2 & No & 15 & Yes & Yes & 0 & Yes \\
\hline 4 & 0.2 & Yes & 12 & Yes & Yes & 0 & No \\
\hline 5 & 0.2 & Yes & 15 & No & Yes & 0.2 & Yes \\
\hline 6 & 0.2 & Yes & 15 & Yes & No & 0.2 & No \\
\hline 7 & 20 & No & 15 & Yes & No & 0 & No \\
\hline 8 & 20 & No & 15 & No & Yes & 0.2 & No \\
\hline 9 & 20 & No & 12 & Yes & Yes & 0.2 & Yes \\
\hline 10 & 20 & Yes & 15 & No & No & 0 & Yes \\
\hline 11 & 20 & Yes & 12 & Yes & No & 0.2 & Yes \\
\hline 12 & 20 & Yes & 12 & No & Yes & 0 & No \\
\hline
\end{tabular}

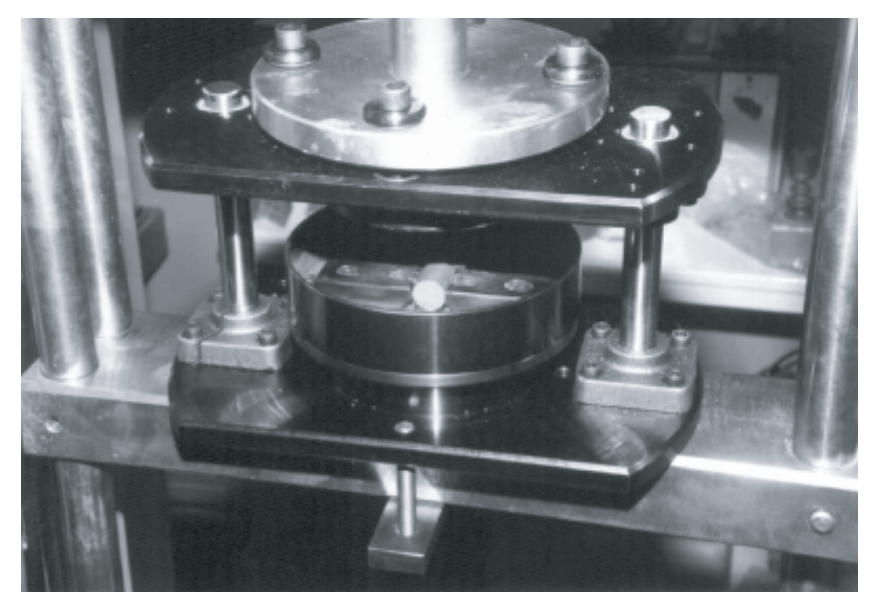

Fig. 4. Experimental device for the diametral compression of massive cylinders.

influences of the material, the sample and the contact between the device and the sample. Namely, these parameters are:

- the cross head speed;

- the sample diameter;
- the sample conicity;

- the surface finishing (presence or no of macro superficial defects introduced by using a diamond tool);

- the geometry of contact: linear or surfacic (samples perfectly cylindrical or provided with two diametrically opposite $1 \mathrm{~mm}$ half flats);

- the nature of sample-device contact (use or no use of two pads ( $0.15 \mathrm{~mm}$ thick) in annealed brass between the sample and the device);

- the action of lateral guides.

\subsubsection{Experimental design results}

The tested samples were pure alumina cylinders of $24 \mathrm{~mm}$ in length. Tests have been carried out in a very short time interval in order to minimise the possible effects induced by variations of the environmental conditions.

The obtained results are illustrated in Figure 5 which shows that:

- the presence of half-flats and the cross head speed play a major role; 


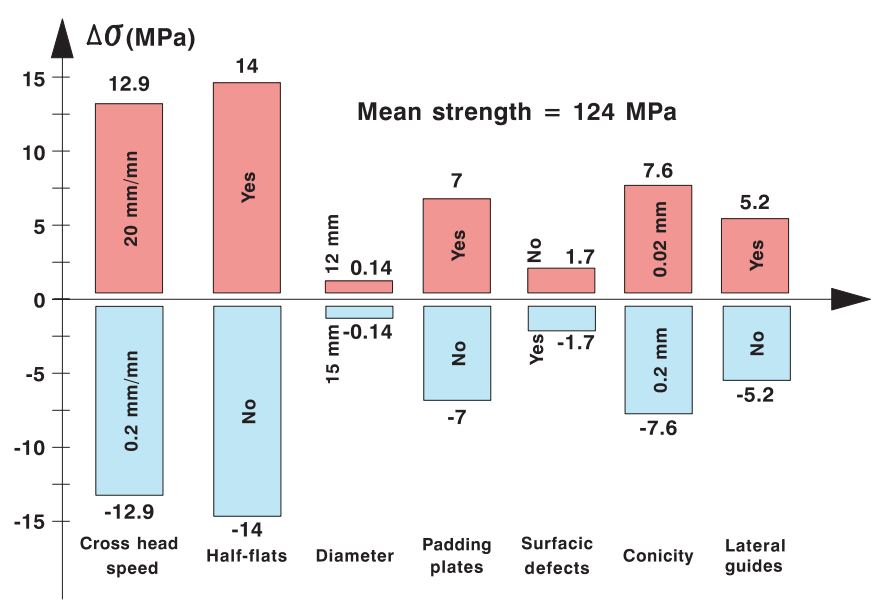

Fig. 5. Experimental design results.

- the sample diameter and the surface finishing play a minor role;

- the sample conicity, the use of padding plates and the effects of lateral guides has an intermediate influence.

It clearly appears that the most important parameter is the presence of half-flats. Indeed, they distribute the applied load and avoid very high contact pressures induced by linear contacts between the sample and the device.

These results are pertinent for the present couple device-material, but it may be thought that some of these influences (i.e. cross head speed) depend on the tested material.

\subsection{Results and discussion}

Finally, the mean strength of tested cylinders can be written as:

$$
\begin{gathered}
\bar{\sigma}_{\mathrm{R}(\mathrm{MPa})}=124+12.9 \delta_{\mathrm{SPEED}}+14 \delta_{\mathrm{FLATS}}+0.14 \delta_{\mathrm{DIAM}} \\
+7 \delta_{\mathrm{PADS}}+1.7 \delta_{\mathrm{DEFECTS}}+7.6 \delta_{\mathrm{CON}}+5.2 \delta_{\mathrm{GUIDES}}
\end{gathered}
$$

with the $\delta$ values given in the Table 2 .

Thus, for cylinders $\Phi 12 \times 24 \mathrm{~mm}$, provided with 2 halfflats of $1 \mathrm{~mm}$ and loaded at $0.2 \mathrm{~mm} \cdot \mathrm{min}^{-1}$ using padding plates, one obtains a mean strength of:

$$
\bar{\sigma}_{\mathrm{R}(\mathrm{MPa})}=124-12.9+14-0.14+7+1.7+7.6+5.2
$$

that is to say about $145 \mathrm{MPa}$.

With bars $\left(4 \times 4 \times 40 \mathrm{~mm}^{3}\right)$ of the same material, loaded in 3 points bending at similar rate, one gets [8] a mean strength of about $250 \mathrm{MPa}$. Starting from this bending value, the tensile strength can be estimated by using the classical result of the 2 parameters Weibull's analysis [9]:

$$
\frac{\sigma_{\mathrm{Flex} 3}}{\sigma_{\mathrm{Tens}}}=\left[2(m+1)^{2}\right]^{1 / m}
$$

$\sigma_{\text {Flex3 }}$ is the 3 point flexural strength, $\sigma_{\text {Tens }}$ the tensile strength and $m$ the Weibull's modulus
Table 2. Values of the $\delta$ coefficients for each case of the experimental design.

\begin{tabular}{|c|c|c|}
\hline Parameter & Value & Coefficient $\delta$ \\
\hline \multirow{2}{*}{ Loading rate } & $20 \mathrm{~mm} \cdot \mathrm{min}^{-1}$ & $\delta_{\text {SPEED }}=1$ \\
\cline { 2 - 3 } & $0.2 \mathrm{~mm} \cdot \mathrm{min}^{-1}$ & $\delta_{\text {SPEED }}=-1$ \\
\hline \multirow{2}{*}{ Half-flats $(1 \mathrm{~mm})$} & Yes & $\delta_{\text {FLATS }}=1$ \\
\cline { 2 - 3 } & No & $\delta_{\text {FLATS }}=-1$ \\
\hline \multirow{2}{*}{ Diameter } & $15 \mathrm{~mm}$ & $\delta_{\text {DIAM }}=1$ \\
\cline { 2 - 3 } & $12 \mathrm{~mm}$ & $\delta_{\text {DIAM }}=-1$ \\
\hline \multirow{2}{*}{ Padding plates } & Yes & $\delta_{\text {PADS }}=1$ \\
\cline { 2 - 3 } & No & $\delta_{\text {PADS }}=-1$ \\
\hline \multirow{2}{*}{ Surfacic defects } & No & $\delta_{\text {DEFECTS }}=1$ \\
\cline { 2 - 3 } & Yes & $\delta_{\text {DEFECTS }}=-1$ \\
\hline \multirow{2}{*}{ Conicity } & $0.02 \mathrm{~mm}$ & $\delta_{\text {CON }}=1$ \\
\cline { 2 - 3 } & $0.2 \mathrm{~mm}$ & $\delta_{\text {CON }}=-1$ \\
\hline \multirow{2}{*}{ Lateral guides } & Yes & $\delta_{\text {GUIDES }}=1$ \\
\cline { 2 - 3 } & No & $\delta_{\text {GUIDES }}=-1$ \\
\hline
\end{tabular}

The tested material having a Weibull's modulus of about 12 , one obtains $\sigma_{\text {Tens }} \approx 154 \mathrm{MPa}$.

Nevertheless, the volume under tensile stresses in the 3 point bending samples being smaller than the volume under the same stresses in the samples subjected to diametral compression, the previous strength must be considered as a upper limiting value of the tensile strength. So, in order to estimate the true tensile strength it would be necessary to perform a correction taking into account the volume effects. Unfortunately, as shown by Marion \& Johnstone [4] such a correction is rather difficult to carry out accurately. However, a limiting lower value can be obtained by considering a flexural sample whose volume under tensile stresses is equal to the whole volume of the compressed sample:

- volume under tensile stresses in 3 point bending samples:

$$
V_{\text {TensBar }}=2 \times 4 \times 40=320 \mathrm{~mm}^{3}
$$

- full volume of cylinders tested in diametral compression:

$$
V_{\mathrm{Cyl}}=\frac{\pi}{4} * 12^{2 *} 24 \approx 2700 \mathrm{~mm}^{3}
$$

- lower limiting tensile stress: $154 *\left[\frac{320}{2700}\right]^{1 / 12} \approx$ $129 \mathrm{MPa}$ (with $m \approx 12$ ).

So, one obtains the following results:

- tensile strength estimated from 3 point bending tests: $129 \mathrm{MPa}<\sigma_{\mathrm{T}}<154 \mathrm{MPa}$,

- tensile strength estimated from diametral compression tests: $\sigma_{\mathrm{T}}=145 \mathrm{MPa}$.

\subsection{Conclusion}

According to bibliographical data [4], this result shows that the test of diametral compression of cylinders, when 
performed with care, is well suited to the estimation of ceramics tensile strength.

However, even when using an improved device, it remains delicate to perform.

\section{Diametral compression of hollow cylinders}

This test is less used than the previous one. However it presents real advantages in the case of ceramic pieces obtained in shape of tubes in which it may be difficult to make bending samples.

Its principle is the same than this of Brazilian test, but it is easier to perform because it requires lower loads.

It leads to two successive fractures of the sample which occur, theoretically, in two perpendicular diametral planes. The first one occurs in the vertical loading plane and starts on the internal surface whereas the second one occurs in the horizontal diametral plane and starts on the external surface. In the ideal case, one obtains 4 perfectly identical sample fragments at the end of the test.

Contrarily to the Brazilian test, the diametral compression of tubes does not give access to tensile but to bending strength.

\subsection{Stress field}

It does not exist a general analytical solution, usable without restriction whatever the ratio "outside diameter/insider diameter" is.

In the particular case where the thickness is weak regarding the outside diameter, the curved beam theory can be applied [10]. In the general case, the numerical test simulation by means of a finite element analysis is the most satisfactory solution. Moreover, it must be noticed that mixed approaches, analytical and numerical, have been proposed [11].

For the two cases of fracture, the numerical simulation leads to stress distributions illustrated by the Figures 6 and 7 .

When the previous tools are not available, or for a rough strength estimation, we propose the following relationship, using the numerical coefficients given in Appendices 1 and 2 .

$$
\sigma_{\mathrm{R}}=\frac{\lambda F}{100 l}
$$

$\sigma_{\mathrm{R}}$ is the material strength (MPa), $F$ the fracture load $(\mathrm{N}), l$ the sample length $(\mathrm{mm})$ and $\lambda$ the coefficient obtained from Appendices 1 or 2 .

These coefficients have been obtained by F.E.A. strength computation (plane strain state) for any diameters combination when $\Phi$ varies from 6 to $30 \mathrm{~mm}$ by step of $1 \mathrm{~mm}$. Generally, actual inside and outside diameters values are not integer. So, when the tube thickness $\left[e=\left(\phi_{\text {ext }}-\phi_{\text {int }}\right) / 2\right]$ is greater than $1 \mathrm{~mm}$, the usable $\lambda$ coefficients can be calculated by linear interpolation between closest $\lambda$ values found in Appendices.

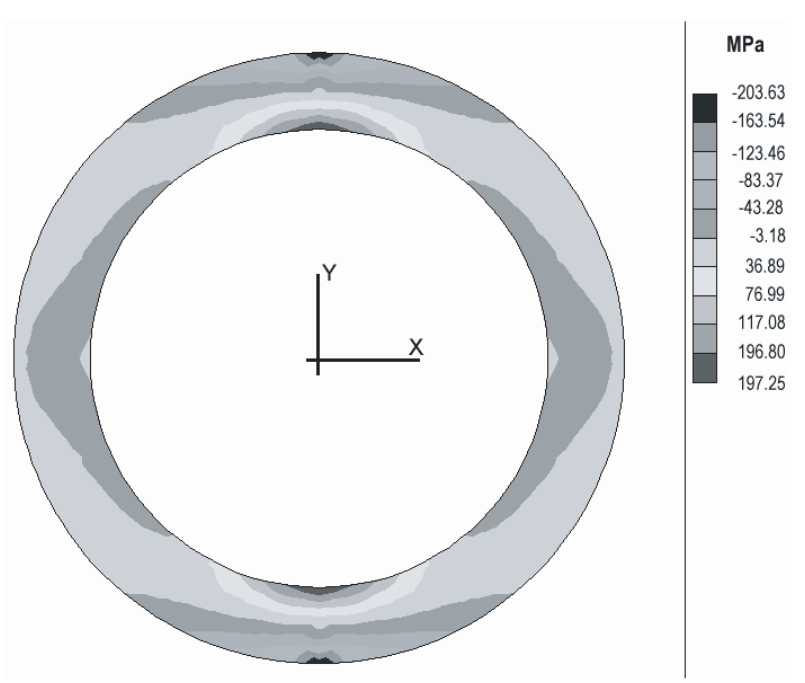

Fig. 6. Rupture in the vertical diametral plane: $\sigma_{X X}$ stresses.

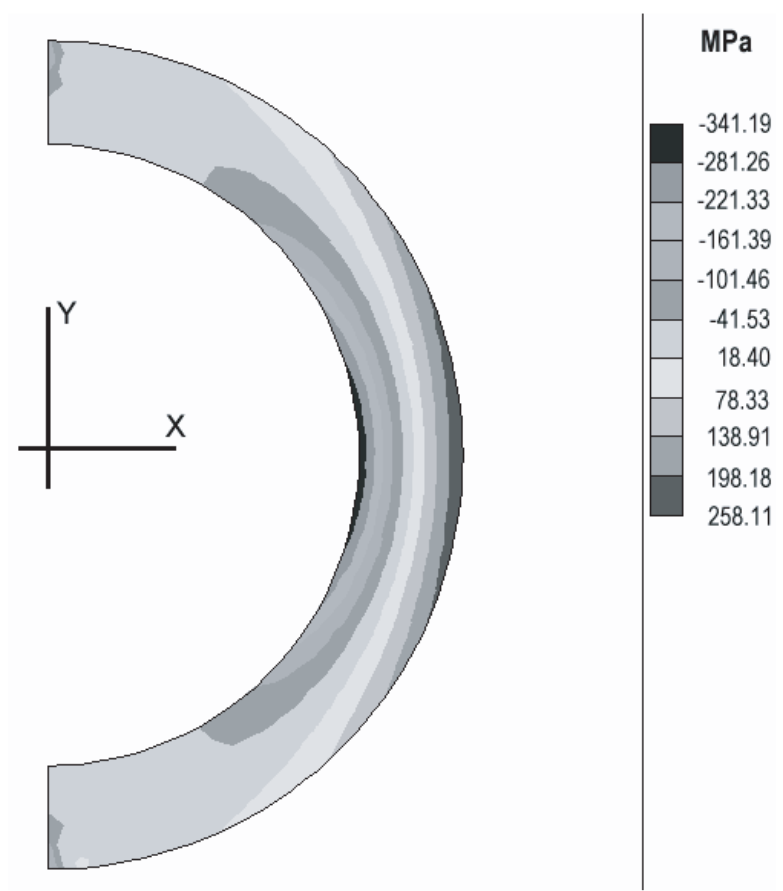

Fig. 7. Rupture in the horizontal diametral plane: $\sigma_{Y Y}$ stresses.

When the tube thickness is lower than this threshold value, the relationships derived from the curved beam theory are usable:

- for fracture occurring in the vertical diameter plane: $\sigma_{\mathrm{R}}=1.92 \frac{F R}{e^{2} l}$

- for fracture occurring in the horizontal diameter plane: $\sigma_{\mathrm{R}}=3 \frac{F R}{e^{2} l}$.

Such calculations lead to the material strength with an error less than 5\% except. However, when fracture occurs in the horizontal diameter plane, the error can reach $10 \%$. Nevertheless, all these calculations can be easily computerised $^{1}$ and, thus, corrections can be taken into

\footnotetext{
1 Such a software is freely available from the first author.
} 

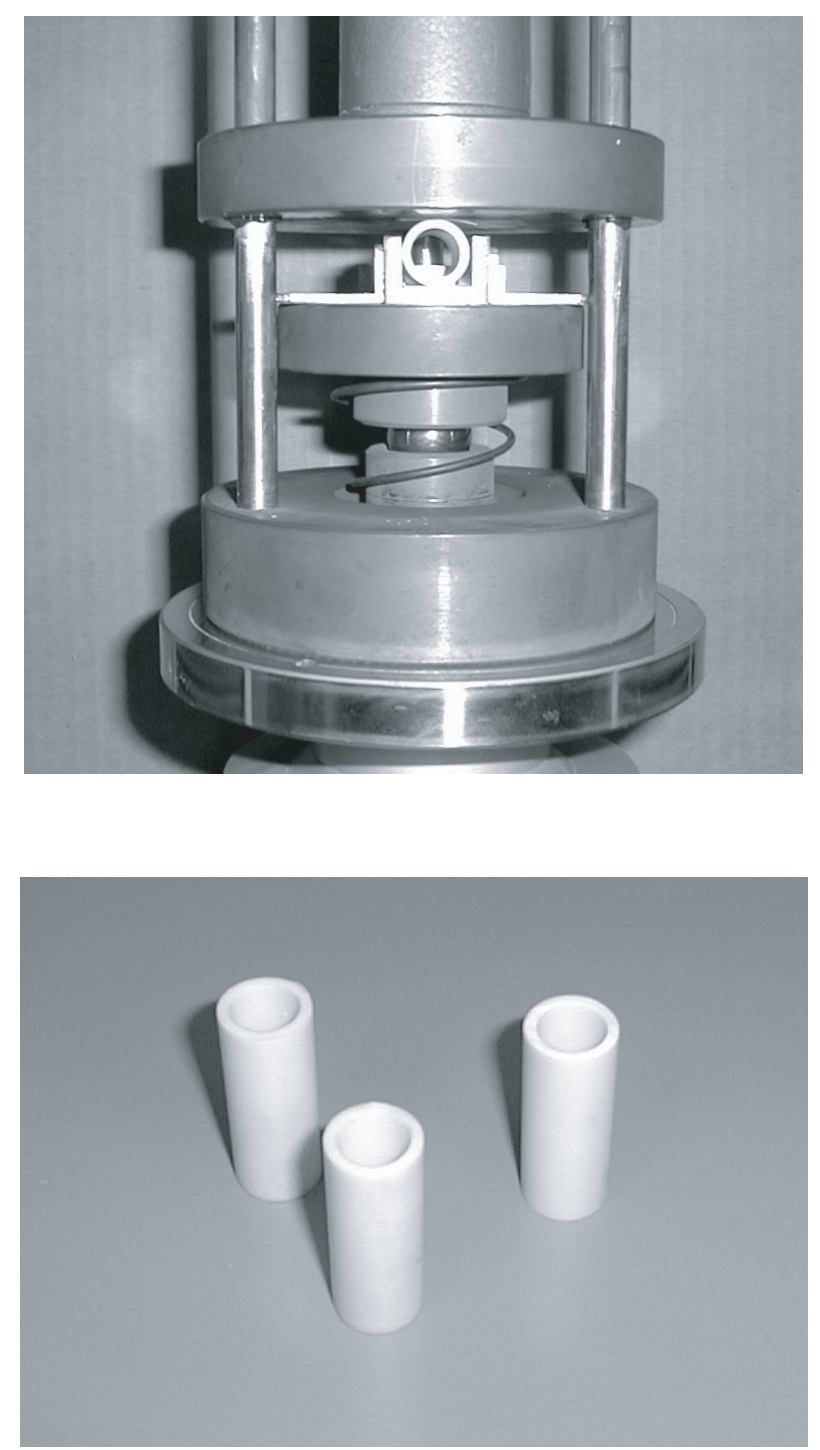

Fig. 8. Experimental device and samples for diametral compression of hollow cylinders.

account to reduce the maximum error to about $5 \%$ whatever the conditions are. Various examples of such computed results are given in Appendix 3.

\subsection{Experimental device and samples}

An experimental device (cf. Fig. 8) has been built on the same principle than this used for massive cylinders: auto alignment of the lower compressive plate maintained in floating position with regard to a spherical support.

The efforts required in this case being significantly lower than those needed by massive cylinders, the lower plate rests on a more compliant elastic support.

The tested samples $(N=15)$ were tubes of commercial pure alumina (Degussa Al23) of $16 \times 12 \mathrm{~mm}$ in diameter and $40 \mathrm{~mm}$ in length.
Tests have been achieved at $0.5 \mathrm{~mm} \cdot \mathrm{min}^{-1}$ cross head

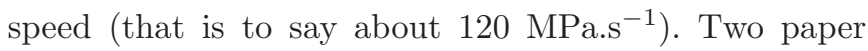
pads ( $0.1 \mathrm{~mm}$ thick) were located between the sample and the compressive plate along the two generating lines of contact. This experimental care is inescapable because it guarantees a good reproducibility of the loading conditions and then leads to significant strength values. To the contrary, if tests are performed without paper pads, fully stochastic fracture occurs and the measured $\sigma_{\mathrm{R}}$ values are too scattered to be considered as meaningful.

\subsection{Results}

The mean strength value reaches $197 \mathrm{MPa}$ when the fracture starts on the internal face of the tube (fracture in the vertical diametral plane) and $258 \mathrm{MPa}$ when it starts on the external face (fracture in the horizontal diametral plane).

These values are in good agreement with the 3 point bending strength measured on bars of the same material, tested at similar loading rate and involving comparable volumes under tensile stresses. Indeed, as previously said $[8,12]$, one obtains $\sigma_{\mathrm{R}}$ values of about $250 \mathrm{MPa}$.

Finally, it can be thought that the difference between the two measured values (197 and $258 \mathrm{MPa}$ ) is due to the difference in the surface finishing between the internal (as sintered) and the external (grinded) surfaces of the tested tubes.

\subsection{Conclusion}

The diametral compression of tubes appears as a technique well suited for the determination of ceramics bending strength. It is easy to perform and needs samples easy to make. However strength values cannot be accurately derived from measured fracture load by means of a simple analytical relationship. The numerical simulation of tests appears as the most convenient method to reach finely this parameter, but the present paper proposes a simple method to perform strength calculation when approximated results are sufficient.

\section{Conclusion}

The ceramic material strength, usually measured by means of 3 or 4 point bending tests, can be determined by diametral compression of cylinders too.

On one hand, the test on massive cylinders is rather delicate to implement and, because it calls for refined experimental devices. However, its optimisation by means of an experimental design permits to obtain meaningful tensile strength values.

On the other hand, the test on hollow cylinders (tubes) does not exhibit major experimental difficulty but the loading data processing calls for the use of stress computation tools (such as finite elements method) able to calculate accurate strength values from the measured 


\begin{tabular}{|c|c|c|c|c|c|c|c|c|c|c|c|c|c|c|c|c|c|c|c|c|c|c|c|c|c|}
\hline m & $\stackrel{m}{\underset{f}{f}}$ & 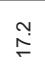 & $\stackrel{\infty}{\infty} \stackrel{\infty}{\stackrel{\infty}{2}}$ & $\stackrel{\infty}{\sim}$ & $\overline{\text { ळ. }}$ & 占 & 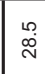 & స్ల & 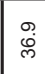 & 号 & $\stackrel{m}{g}$ & $\begin{array}{c}0 \\
0 \\
0 \\
w\end{array}$ & & 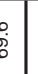 & డ్ & 号 & 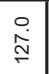 & 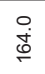 & & 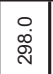 & 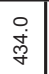 & $\begin{array}{l}0 \\
0 \\
0 \\
0\end{array}$ & 品 & 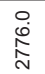 & \\
\hline శ్ & $\widehat{\grave{g}}$ & 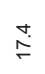 & $\stackrel{g}{\stackrel{g}{\sigma}}$ & $\stackrel{\mathscr{N}}{ }$ & $\stackrel{\infty}{\stackrel{\infty}{ \pm}}$ & $\underset{N}{\stackrel{N}{N}}$ & 官 & 兄 & $\stackrel{\circ}{\dot{\sigma}}$ & 等 & 苞 & 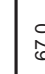 & & ?he & $\begin{array}{l}\infty \\
\stackrel{\infty}{\sigma} \\
\end{array}$ & స్ ָָ & $\begin{array}{l}\text { 足 } \\
\stackrel{\leftrightarrow}{\circ}\end{array}$ & 品 & 足 & 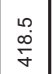 & 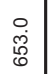 & 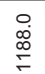 & 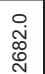 & $\begin{array}{l}\text { 吕 } \\
\text { d. } \\
0.0\end{array}$ & 1 \\
\hline ๙ & $\stackrel{0}{r}$ & $\begin{array}{l}\stackrel{\infty}{\infty} \\
\stackrel{\infty}{\infty}\end{array}$ & $\underset{⿱ 亠}{\stackrel{*}{~}}$ & 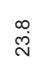 & $\stackrel{m}{i}$ & 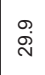 & $\mid \begin{array}{c}\text { f } \\
\text { ja }\end{array}$ & 芦 & $\mid \hat{q}$ & 管 & 童 & co & & 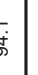 & 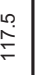 & فે & 宫 & 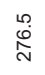 & 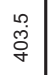 & ণ্ণ் & 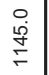 & $\begin{array}{l}0 \\
\infty \\
\infty \\
\text { N }\end{array}$ & $\begin{array}{l}0 \\
0 \\
0 \\
0 \\
0\end{array}$ & 1 & 1 \\
\hline$\hat{\sim}$ & $\stackrel{\circ}{\stackrel{\infty}{\infty}}$ & 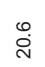 & ָे & $\underset{\dot{I}}{\bar{I}}$ & $\begin{array}{l}\infty \\
\stackrel{\infty}{\infty} \\
\sim\end{array}$ & ఖ్లె & \begin{tabular}{|l}
$\infty$ \\
$\stackrel{m}{m}$
\end{tabular} & $\begin{array}{l}\dot{g} \\
\dot{\gamma}\end{array}$ & نำ & $\stackrel{\infty}{\overline{0}}$ & $\stackrel{m}{i}_{i}$ & : & & $\stackrel{\rho}{b}$ & 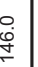 & 怘 & 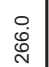 & 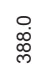 & $\begin{array}{l}0 \\
\dot{8} \\
8\end{array}$ & 㒸 & 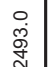 & 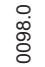 & 1 & 1 & 1 \\
\hline$\stackrel{\overbrace{}}{\sim}$ & Ф̊) & $\stackrel{\sigma}{\dot{N}}$ & 롤 & $\stackrel{\circ}{\sim}$ & $\stackrel{\text { po }}{\bar{m}}$ & ్ֶָల & $\overline{\mathcal{J}}$ & $\begin{array}{l}\infty \\
\dot{\sigma} \\
\dot{\sigma}\end{array}$ & 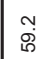 & $\stackrel{m}{i}$ & 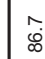 & 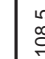 & & 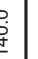 & 幽 & 葛串 & 足 & 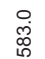 & $\begin{array}{l}\circ \\
: \\
0\end{array}$ & $\begin{array}{l}\circ \\
\stackrel{8}{0} \\
\text { o. }\end{array}$ & 品 & 1 & 1 & 1 & 1 \\
\hline$\stackrel{\sim}{\sim}$ & $\stackrel{\circ}{\stackrel{一}{N}}$ & 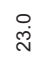 & 号 & 岂 & fi & $\stackrel{m}{\stackrel{q}{q}}$ & \begin{tabular}{|l}
$\stackrel{\leftrightarrow}{\sim}$ \\
$\dot{\gamma}$
\end{tabular} & $\begin{array}{l}\text { : } \\
\stackrel{\circ}{\circ}\end{array}$ & : & $\stackrel{\infty}{\infty}$ & 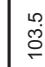 & 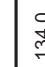 & & ? & 葛 & 足 & 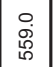 & 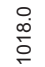 & 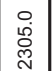 & 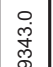 & 1 & 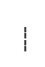 & 1 & 1 & 1 \\
\hline ‡ & $\stackrel{9}{\dot{N}}$ & బ్ల & 餇 & 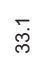 & 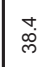 & 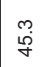 & 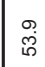 & $\frac{m}{\dot{d}}$ & 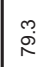 & $\stackrel{\infty}{\infty}$ & & $\begin{array}{l}5 \\
\vdots \\
0 \\
0\end{array}$ & & 总 & 旤 & $\begin{array}{l}\text { 它 } \\
\text { in }\end{array}$ & 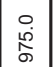 & $\begin{array}{l}\text { P. } \\
\stackrel{\tilde{N}}{\tilde{N}}\end{array}$ & $\begin{array}{l}0 \\
\stackrel{0}{0} \\
0 \\
\infty\end{array}$ & 1 & 1 & ! & 1 & 1 & 1 \\
\hline ָ & $\stackrel{\text { ָे }}{\text { N }}$ & 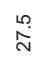 & $\stackrel{n}{p}$ & 迎 & $\overline{\dot{g}}$ & $\stackrel{m}{\dot{\omega}}$ & $\frac{m}{\dot{\sigma}}$ & 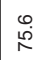 & \begin{tabular}{|l} 
Fे \\
\end{tabular} & ্ָ & తి & $\stackrel{n}{n}$ & & : & 离 & 总 & 号 & $\begin{array}{l}\text { 車 } \\
\text { on }\end{array}$ & 1 & 1 & 1 & 1 & 1 & 1 & 1 \\
\hline$\approx$ & 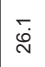 & 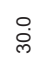 & $\begin{array}{l}\infty \\
\dot{m} \\
\dot{m}\end{array}$ & $\begin{array}{l}\dot{\sigma} \\
\dot{\sigma}\end{array}$ & 䢵 & $\underset{\infty}{\infty}$ & $\underset{\mid \stackrel{g}{r}}{\stackrel{\rho}{r}}$ & 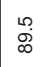 & 㒸 & 先 & 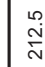 & $\frac{\rho}{\bar{m}}$ & & 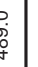 & $\begin{array}{l}8 \\
\\
\end{array}$ & ๙్்ָ & 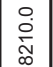 & 1 & 1 & 1 & 1 & 1 & 1 & 1 & 1 \\
\hline $\bar{\wedge}$ & $\underset{\sim}{\grave{D}}$ & 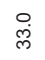 & 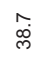 & $\bar{g}$ & 总 & 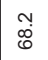 & 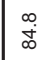 & 嗛 & 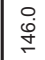 & ฌ્ं & @্ & 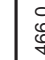 & & $\begin{array}{l}0 \\
0 \\
0 \\
0\end{array}$ & 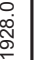 & $\begin{array}{l}\text { 总 } \\
\text { o. }\end{array}$ & 1 & 1 & 1 & 1 & 1 & 1 & 1 & 1 & 1 \\
\hline ণ & ָू & 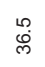 & $\begin{array}{l}\text { 吕 } \\
\text { ro }\end{array}$ & $\underset{\overbrace{}}{~}$ & 赵 & $\overline{\dot{\infty}}$ & 过 & 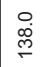 & 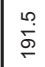 & 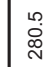 & 岁 & 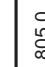 & & 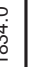 & 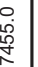 & 1 & 1 & 1 & 1 & 1 & 1 & 1 & 1 & 1 & 1 \\
\hline$\stackrel{9}{\circ}$ & $\stackrel{m}{\tilde{j}}$ & 字 & ஜூj & $\stackrel{\infty}{\circ} \stackrel{0}{0}$ & 芯 & $\stackrel{\dot{\sigma}}{\tilde{\sigma}}$ & 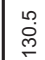 & 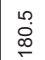 & 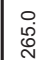 & $\begin{array}{l}\frac{0}{\alpha} \\
\frac{j}{7}\end{array}$ & : & 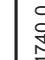 & & $\frac{0}{5}$ & | & 1 & 1 & 1 & 1 & 1 & 1 & 1 & 1 & 1 & 1 \\
\hline 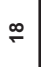 & $\underset{\substack{\infty \\
\infty}}{ }$ & 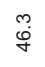 & ت્ّ & $\stackrel{\infty}{\stackrel{\infty}{R}}$ & \begin{tabular}{|l}
$\dot{2}$ \\
$\dot{\sigma}$
\end{tabular} & ָ̊ & 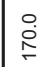 & 吕 & 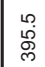 & ্ㅗ & 量 & cे & & | & | & 1 & 1 & 1 & 1 & 1 & 1 & 1 & 1 & 1 & 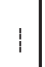 \\
\hline$=$ & $\underset{\mathscr{g}}{\stackrel{m}{\sigma}}$ & 芦 & $\bar{\phi}$ & $\underset{\infty}{\infty}$ & 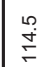 & $\begin{array}{l}\circ \\
\stackrel{8}{\circ}\end{array}$ & 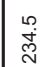 & ్ㅗㄹ & \begin{tabular}{|l}
0 \\
0 \\
0 \\
0
\end{tabular} & 总 & 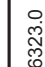 & 1 & & ; & 1 & 1 & 1 & 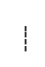 & 1 & 1 & 1 & 1 & 1 & 1 & 1 \\
\hline$\stackrel{\circ}{\circ}$ & 产 & $\frac{\dot{\sigma}}{\sigma}$ & 寄 & $\begin{array}{l}\text { 足 } \\
\stackrel{8}{\&}\end{array}$ & 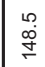 & 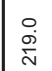 & $\begin{array}{l}\stackrel{\circ}{\circ} \\
\stackrel{j}{j}\end{array}$ & $\begin{array}{l}0 \\
\text { i. } \\
\text { i. }\end{array}$ & 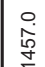 & 市 & 1 & 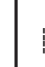 & & 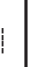 & 1 & ! & 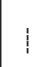 & 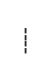 & 1 & 1 & 1 & 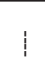 & 1 & 1 & 1 \\
\hline 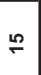 & $\begin{array}{l}\widehat{0} \\
\stackrel{6}{b}\end{array}$ & $\stackrel{\infty}{i}$ & $\begin{array}{l}\infty \\
\infty \\
\infty \\
\infty\end{array}$ & $\begin{array}{l}\text { o. } \\
\stackrel{c}{c}\end{array}$ & | & 怘 & 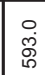 & $\begin{array}{l}\text { : } \\
\text { : }\end{array}$ & 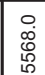 & 1 & 1 & 1 & & 1 & 1 & 1 & 1 & 1 & 1 & 1 & 1 & 1 & 1 & 1 & 1 \\
\hline \pm & $\stackrel{\mathscr{\theta}}{\tilde{\theta}}$ & ㅎ. & 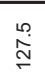 & 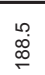 & ه্ं & 品 & 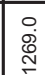 & 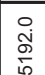 & 1 & 1 & 1 & 1 & & 1 & 1 & 1 & 1 & 1 & 1 & 1 & 1 & 1 & 1 & 1 & 1 \\
\hline$\stackrel{m}{m}$ & $\bar{\infty}$ & 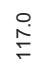 & 昂 & 足 & 苘 & 惫 & 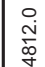 & 1 & 1 & 1 & 1 & 1 & & | & 1 & 1 & 1 & 1 & 1 & 1 & 1 & 1 & 1 & 1 & 1 \\
\hline$\cong$ & 迥 & 吕 & 芯 & $\begin{array}{l}\text { o. } \\
\text { ga }\end{array}$ & 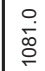 & 畹 & 1 & 1 & 1 & 1 & 1 & 1 & & 1 & 1 & 1 & 1 & 1 & 1 & 1 & 1 & 1 & 1 & 1 & 1 \\
\hline$=$ & q্ & 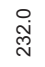 & 吕 & $\begin{array}{l}\stackrel{\circ}{\circ} \\
\infty\end{array}$ & 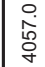 & 1 & 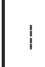 & 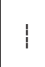 & 1 & 1 & 1 & 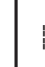 & & 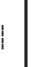 & | & 1 & 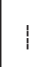 & 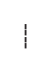 & 1 & 1 & 1 & 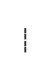 & 1 & 1 & 1 \\
\hline$\therefore$ & $\begin{array}{l}\stackrel{0}{\circ} \stackrel{0}{\stackrel{0}{\sim}}\end{array}$ & $\underset{\substack{\dot{m} \\
\text { ’ }}}{ }$ & 尺̊. & 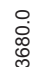 & 1 & 1 & 1 & 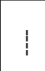 & 1 & 1 & 1 & 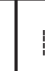 & & ; & 1 & 1 & 1 & 1 & 1 & 1 & 1 & 1 & 1 & 1 & 1 \\
\hline の & 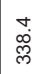 & 足 & 总 & 1 & 1 & 1 & 1 & 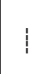 & 1 & 1 & 1 & 1 & & | & 1 & 1 & 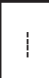 & 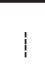 & 1 & 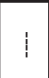 & 1 & 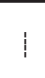 & 1 & 1 & 1 \\
\hline$\infty$ & 号 & 岕 & 1 & 1 & 1 & 1 & 1 & 1 & 1 & 1 & | & 1 & & ; & 1 & 1 & 1 & 1 & 1 & 1 & 1 & 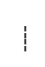 & 1 & 1 & 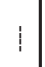 \\
\hline N & 号嚆 & 1 & 1 & 1 & 1 & 1 & 1 & 1 & 1 & 1 & 1 & 1 & & 1 & 1 & 1 & 1 & 1 & 1 & 1 & 1 & 1 & 1 & 1 & 1 \\
\hline & 0 & r & $\infty$ & 0 & $\therefore$ & $=$ & $\cong$ & $\stackrel{m}{q}$ & \pm & $\cong$ & $\stackrel{\circ}{\circ}$ & & & 0 & & ণ & $\bar{N}$ & N & $\approx$ & I & $\stackrel{\sim}{N}$ & $\stackrel{\text { N }}{\circ}$ & $\hat{N}$ & $\stackrel{\infty}{\sim}$ & న \\
\hline
\end{tabular}




\begin{tabular}{|c|c|c|c|c|c|c|c|c|c|c|c|c|c|c|c|c|c|c|c|c|c|c|c|c|}
\hline 官 & $\bar{\sigma}$ & $\stackrel{\hat{\rho}}{\dot{b}}$ & 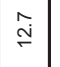 & 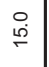 & $\stackrel{\infty}{\stackrel{2}{\sim}}$ & $\overline{\dot{N}}$ & 灾 & 离 & 怘 & \begin{tabular}{|l}
$\stackrel{\circ}{q}$ \\
$\dot{q}$
\end{tabular} & ণે & \begin{tabular}{l}
0 \\
0 \\
\hdashline
\end{tabular} & $\begin{array}{l}\substack{\infty \\
i} \\
\end{array}$ & $\mid$\begin{tabular}{l}
\multirow{2}{*}{} \\
$\vdots$
\end{tabular} & $\stackrel{\text { 足 }}{\stackrel{\sim}{\sim}}$ & 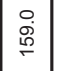 & $\begin{array}{l}\stackrel{\circ}{\circ} \\
\stackrel{\circ}{N}\end{array}$ & 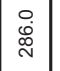 & 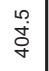 & $\left|\begin{array}{l}0 \\
0 \\
0 \\
0\end{array}\right|$ & 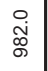 & $\mid \begin{array}{l}0 \\
\stackrel{0}{0} \\
\stackrel{0}{\infty}\end{array}$ & 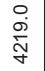 & 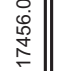 \\
\hline$\stackrel{\mathrm{j}}{\mathrm{j}}$ & $\hat{\sigma}$ & $\begin{array}{l}\stackrel{\circ}{\circ} \\
\stackrel{2}{*}\end{array}$ & $\stackrel{\widehat{\rho}}{\stackrel{\rho}{g}}$ & 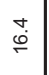 & $\stackrel{\circ}{\stackrel{\circ}{\sigma}}$ & $\stackrel{+}{\stackrel{\sim}{~}}$ & 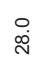 & 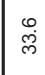 & 苟 & $\mid \bar{q}$ & $\overline{8}$ & \begin{tabular}{l}
$\mathfrak{Y}$ \\
\multirow{Z}{Z}{}
\end{tabular} & 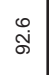 & 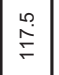 & 宊 & $\mid \begin{array}{l}0 \\
\dot{\dot{a}} \\
\end{array}$ & 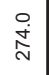 & $\begin{array}{l}0 \\
\infty \\
\infty \\
\infty \\
\infty\end{array}$ & 品 & \begin{tabular}{|l}
0 \\
$\dot{j}$ \\
$\dot{j}$
\end{tabular} & 兽 & $\begin{array}{l}0 \\
\stackrel{0}{0} \\
o ̛ g\end{array}$ & 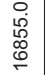 & 1 \\
\hline$\stackrel{\circ}{\text { ì }}$ & 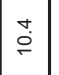 & 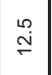 & $\left|\begin{array}{l}0 \\
\dot{\rho} \\
\dot{\rho}\end{array}\right|$ & $\stackrel{\circ}{\stackrel{\infty}{\infty}}$ & $\mid \begin{array}{l}0 \\
\stackrel{\sim}{N}\end{array}$ & $\stackrel{\circ}{\stackrel{\leftrightarrow}{N}}$ & $\stackrel{m}{\dot{m}}$ & 离 & $\bar{g}$ & $\mid \begin{array}{l}0 \\
\stackrel{0}{0} \\
6\end{array}$ & $\bar{i}$ & $\mid$\begin{tabular}{c|}
$\infty$ \\
$\stackrel{\infty}{\infty}$ \\
$\infty$
\end{tabular} & 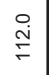 & $\mid \begin{array}{l}0 \\
\dot{f} \\
\end{array}$ & $\begin{array}{l}\text { 吕 } \\
\stackrel{\sigma}{\sigma}\end{array}$ & 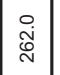 & 年 & $\left|\begin{array}{l}0 \\
\dot{0} \\
0 \\
0\end{array}\right|$ & $\begin{array}{l}0 \\
\dot{\tilde{O}} \\
\dot{a}\end{array}$ & 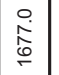 & $\begin{array}{l}\text { 品 } \\
\bar{\delta} \\
\end{array}$ & 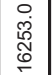 & 1 & 1 \\
\hline$\stackrel{i}{i}$ & $\stackrel{\stackrel{P}{\perp}}{=}$ & $\begin{array}{l}\stackrel{\circ}{\stackrel{\rho}{~}} \\
\end{array}$ & $\begin{array}{l} \pm \\
\stackrel{0}{\circ}\end{array}$ & 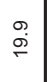 & $\stackrel{\circ}{\stackrel{N}{N}}$ & হं & 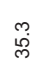 & \begin{tabular}{|l}
$\tilde{y}$ \\
$\tilde{\gamma}$
\end{tabular} & 记 & $\overline{\dot{\theta}}$ & 嗢 & $\mid$\begin{tabular}{l}
0 \\
$\stackrel{0}{0}$ \\
\hdashline
\end{tabular} & \begin{tabular}{|l}
$\stackrel{0}{0}$ \\
$\stackrel{\rho}{0}$
\end{tabular} & $\mid \begin{array}{c}\underset{j}{\infty} \\
\stackrel{\infty}{\sim}\end{array}$ & 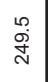 & 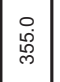 & $\begin{array}{l}\text { 号 } \\
\text { 总 }\end{array}$ & $\left|\begin{array}{l}0 \\
\dot{0} \\
0 \\
\infty\end{array}\right|$ & $\begin{array}{l}0 \\
\stackrel{0}{0} \\
\stackrel{0}{0}\end{array}$ & $\begin{array}{l}0 \\
0 \\
0 \\
0 \\
0\end{array}$ & $\begin{array}{l}\text { : } \\
\text { : } \\
\text { d. }\end{array}$ & 1 & 1 & 1 \\
\hline 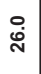 & $\stackrel{\sim}{\sim}$ & $\stackrel{\mathscr{P}}{\dot{I}}$ & $\stackrel{\bar{\infty}}{=}$ & $\overline{\mathrm{i}}$ & 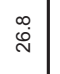 & $\stackrel{\infty}{\underset{ల}{~}}$ & $\stackrel{N}{G}$ & \begin{tabular}{|l}
$\infty$ \\
$\dot{j}$ \\
$\dot{\sigma}$
\end{tabular} & ণ્ণి & $\begin{array}{l}\bar{\infty} \\
\stackrel{\infty}{\sim}\end{array}$ & 界 & \begin{tabular}{|l|}
$\stackrel{\circ}{\circ}$ \\
$\stackrel{p}{\circ}$
\end{tabular} & 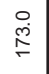 & $\mid \begin{array}{c}\stackrel{0}{0} \\
\stackrel{\tilde{N}}{N}\end{array}$ & 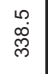 & $\mid \begin{array}{l}0 \\
0 \\
0 \\
i n\end{array}$ & 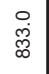 & 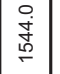 & $\begin{array}{l}0 \\
0 \\
0 \\
0 \\
0 \\
0\end{array}$ & 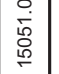 & 1 & 1 & 1 & 1 \\
\hline 埅 & $\mid \begin{array}{l}\infty \\
\stackrel{\infty}{\infty}\end{array}$ & 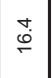 & $\bar{i}$ & 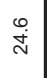 & $\begin{array}{l}\text { N } \\
\stackrel{\rho}{0}\end{array}$ & 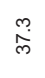 & $\underset{\substack{0 \\
\stackrel{g}{0}}}{ }$ & 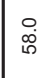 & $m_{n}^{\infty}$ & 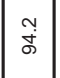 & 总 & $\mid \begin{array}{l}0 \\
\dot{f} \\
0 \\
0\end{array}$ & 乫 & 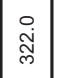 & 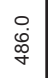 & $\mid$\begin{tabular}{|l|}
0 \\
0 \\
0 \\
0
\end{tabular} & 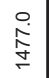 & $\left|\begin{array}{|c}0 \\
0 \\
0 \\
0 \\
0\end{array}\right|$ & $\begin{array}{l}\text { 守 } \\
\stackrel{+}{+}\end{array}$ & 1 & 1 & 1 & 1 & 1 \\
\hline 守 & $\mid \begin{array}{l}0 \\
\dot{\Phi} \\
\end{array}$ & $\stackrel{\bar{\infty}}{\stackrel{\infty}{\infty}}$ & $\stackrel{\infty}{\sim}$ & $\hat{i}$ & $\begin{array}{l}+ \\
\dot{D} \\
\end{array}$ & 守 & 品 & 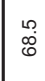 & $\underset{\infty}{\infty}$ & 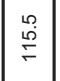 & 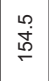 & 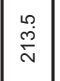 & $\begin{array}{l}\text { 吕 } \\
\text { 怘 }\end{array}$ & 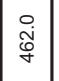 & $\begin{array}{l}\text { 足 } \\
\text { 足 }\end{array}$ & $\left|\begin{array}{l}0 \\
\dot{0} \\
\vdots \\
\dot{y}\end{array}\right|$ & 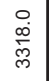 & 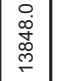 & 1 & 1 & 1 & 1 & 1 & 1 \\
\hline$\stackrel{\stackrel{\sim}{j}}{\dot{j}}$ & $\mid \overline{\dot{\phi}}$ & $\overline{\dot{i}}$ & 灾 & $\stackrel{+}{\dot{m}}$ & 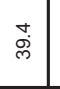 & 字 & $\hat{\mathscr{c}}$ & $\underset{\infty}{\stackrel{\infty}{\sim}}$ & 兽品 & 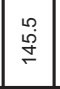 & 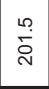 & 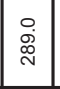 & 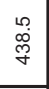 & $\mid \stackrel{0}{\dot{i}}$ & 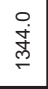 & \begin{tabular}{|l|} 
\\
$\stackrel{0}{0}$ \\
$\dot{m}$ \\
\end{tabular} & 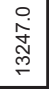 & 1 & 1 & 1 & 1 & 1 & 1 & 1 \\
\hline নं & $\begin{array}{l}\stackrel{\infty}{\sim} \\
\sim\end{array}$ & $\stackrel{\circ}{\mathcal{N}}$ & $\left|\begin{array}{l}\infty \\
\infty \\
\infty \\
\infty\end{array}\right|$ & $\begin{array}{l}\stackrel{0}{0} \\
\bar{\infty}\end{array}$ & 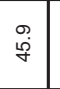 & 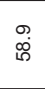 & 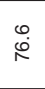 & |옿 & $\begin{array}{l}\text { 吕 } \\
\stackrel{\rho}{\sigma}\end{array}$ & \begin{tabular}{|l|}
$\stackrel{0}{0}$ \\
$\stackrel{\infty}{\circ}$
\end{tabular} & 足 & 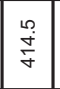 & 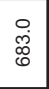 & 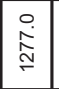 & $\begin{array}{l}0 \\
\stackrel{0}{0} \\
\dot{\tilde{n}}\end{array}$ & 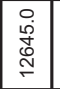 & 1 & 1 & 1 & 1 & 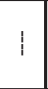 & 1 & 1 & 1 \\
\hline$\stackrel{0}{\dot{\lambda}}$ & $\stackrel{\circ}{\dot{D}}$ & 点 & 心్ల & $\begin{array}{l}\infty \\
\stackrel{\infty}{\sigma}\end{array}$ & 要 & $\hat{i}$ & 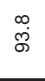 & 突 & 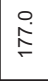 & 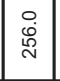 & \begin{tabular}{|l} 
\\
$\stackrel{\circ}{0}$ \\
0 \\
\end{tabular} & 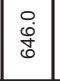 & 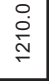 & 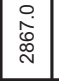 & \begin{tabular}{l} 
姜 \\
\multirow{2}{*}{}
\end{tabular} & 1 & 1 & 1 & 1 & 1 & 1 & 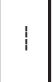 & 1 & 1 \\
\hline$\stackrel{\text { i }}{\text { in }}$ & 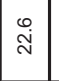 & $\overline{\dot{\sigma}}$ & $\begin{array}{l}\stackrel{\infty}{0} \\
\stackrel{m}{m}\end{array}$ & $\underset{\tilde{g}}{\tilde{q}}$ & $\begin{array}{l}\mathscr{P} \\
\dot{0}\end{array}$ & 怘 & $\stackrel{\stackrel{\infty}{\circ}}{\stackrel{0}{=}}$ & \begin{tabular}{|l}
$\stackrel{\circ}{\circ}$ \\
$\stackrel{\leftrightarrow}{\circ}$
\end{tabular} & 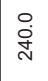 & \begin{tabular}{|l}
0 \\
0 \\
0
\end{tabular} & $\begin{array}{l}0 \\
0 \\
8 \\
0\end{array}$ & 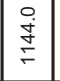 & $\begin{array}{l}0 \\
\stackrel{2}{1} \\
\text { N. }\end{array}$ & 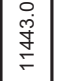 & 1 & 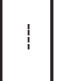 & 1 & 1 & 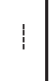 & 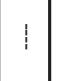 & 1 & 1 & 1 & 1 \\
\hline$\stackrel{\circ}{\stackrel{\circ}{\sigma}}$ & $\stackrel{\hat{\rho}}{\sim}$ & 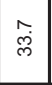 & 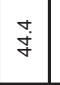 & 品 & $\begin{array}{l}\infty \\
\rho \\
i\end{array}$ & $\begin{array}{l}\text { 足 } \\
\stackrel{\infty}{0}\end{array}$ & 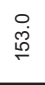 & 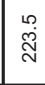 & 品 & \begin{tabular}{|l|l}
$\stackrel{0}{\dot{B}}$ \\
$\dot{i}$
\end{tabular} & 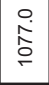 & 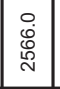 & 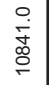 & 1 & 1 & 1 & 1 & 1 & 1 & 1 & 1 & 1 & 1 & 1 \\
\hline$\stackrel{\circ}{\stackrel{\circ}{\sim}}$ & $\hat{\stackrel{a}{\sim}}$ & $\begin{array}{l}\circ \\
\stackrel{\circ}{\circ}\end{array}$ & 总 & 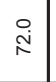 & $\mid \begin{array}{l}\infty \\
\stackrel{\infty}{\sigma}\end{array}$ & 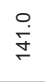 & 定 & \begin{tabular}{|l}
$\frac{O}{\rho}$ \\
$\dot{m}$
\end{tabular} & 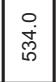 & $\mid$\begin{tabular}{|l|}
$\stackrel{0}{\dot{亠}}$ \\
$\dot{\bar{c}}$
\end{tabular} & 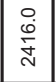 & 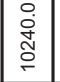 & 1 & 1 & 1 & 1 & 1 & 1 & 1 & 1 & 1 & 1 & 1 & 1 \\
\hline 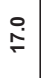 & $\begin{array}{l}\infty \\
\dot{m} \\
\dot{m}\end{array}$ & $\begin{array}{l}\mathscr{N} \\
\tilde{\gamma}\end{array}$ & $\begin{array}{l}\infty \\
\dot{0} \\
0\end{array}$ & $\begin{array}{l}n \\
\check{\circ}\end{array}$ & $\begin{array}{l}0 \\
\stackrel{N}{\sim} \\
\sim\end{array}$ & 怘 & $\begin{array}{l}\text { 另 } \\
\stackrel{\leftrightarrow}{\mathrm{N}}\end{array}$ & 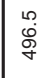 & $\begin{array}{l}0 \\
j \\
⿱\end{array}$ & 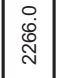 & 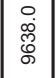 & 1 & 1 & 1 & 1 & 1 & 1 & 1 & 1 & 1 & 1 & 1 & 1 & 1 \\
\hline $\begin{array}{l}\stackrel{\circ}{\dot{\phi}} \\
\end{array}$ & $\frac{d}{\dot{J}}$ & 旡 & $\stackrel{0}{\dot{\infty}}$ & $\begin{array}{l}\stackrel{\ell}{\circ} \\
\stackrel{0}{\leftarrow} \\
\end{array}$ & 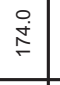 & 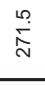 & 吕客 & \begin{tabular}{|l}
$\stackrel{0}{0}$ \\
$\hat{\infty}$
\end{tabular} & $\begin{array}{l}0 \\
\stackrel{0}{N} \\
\end{array}$ & 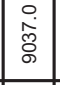 & 1 & 1 & 1 & 1 & 1 & 1 & 1 & 1 & 1 & 1 & 1 & 1 & 1 & 1 \\
\hline$\stackrel{0}{\stackrel{0}{\rho}}$ & $\left|\begin{array}{c}m \\
0 \\
i \\
0\end{array}\right|$ & $\stackrel{\infty}{\stackrel{\infty}{\wedge}}$ & $\begin{array}{l}0 \\
\stackrel{0}{0} \\
\stackrel{0}{0}\end{array}$ & 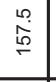 & 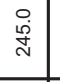 & ঙ్̊ㄱ & $\underset{\infty}{\stackrel{\circ}{\dot{亠}}}$ & \begin{tabular}{|l}
$\stackrel{\circ}{\circ}$ \\
$\stackrel{8}{\circ}$
\end{tabular} & 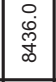 & 1 & 1 & 1 & 1 & 1 & 1 & 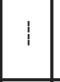 & 1 & 1 & 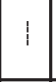 & 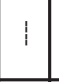 & 1 & 1 & 1 & 1 \\
\hline$\stackrel{O}{\dot{f}}$ & $\begin{array}{l}\mathscr{D} \\
\mathscr{\delta}\end{array}$ & $\mid \begin{array}{l}\mathscr{J} \\
\sigma\end{array}$ & $\begin{array}{l}\stackrel{0}{\dot{\Phi}} \\
\dot{5}\end{array}$ & 品 & 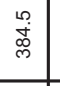 & 亭 & 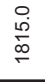 & 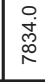 & 1 & 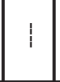 & 1 & 1 & 1 & 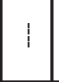 & 1 & 1 & 1 & 1 & 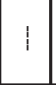 & 1 & 1 & 1 & 1 & 1 \\
\hline$\stackrel{\circ}{\stackrel{\rho}{\stackrel{\rho}{r}}}$ & $\mid \begin{array}{l} \\
\stackrel{\infty}{0}\end{array}$ & $\mid \begin{array}{l}0 \\
\stackrel{0}{ \pm} \\
\end{array}$ & $\mid \begin{array}{l}0 \\
\dot{0} \\
\dot{\sim}\end{array}$ & $\begin{array}{l}0 \\
\dot{y} \\
\dot{y}\end{array}$ & $\left|\begin{array}{l}0 \\
0 \\
0 \\
0\end{array}\right|$ & $\begin{array}{l}\text { 吕 } \\
\stackrel{\leftrightarrow}{\circ}\end{array}$ & 怨 & 1 & 1 & 1 & 1 & 1 & 1 & 1 & 1 & 1 & 1 & 1 & 1 & 1 & 1 & 1 & 1 & 1 \\
\hline 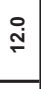 & $\mid \begin{array}{l}0 \\
\stackrel{0}{\circ} \\
\stackrel{0}{0}\end{array}$ & \begin{tabular}{|l|}
$\stackrel{\leftrightarrow}{\circ}$ \\
$\stackrel{0}{\circ}$
\end{tabular} & $\mid \begin{array}{l}0 \\
\stackrel{0}{0} \\
\end{array}$ & $\stackrel{\circ}{\frac{1}{0}}$ & 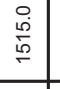 & 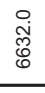 & 1 & 1 & 1 & 1 & 1 & 1 & 1 & 1 & 1 & 1 & 1 & 1 & 1 & 1 & 1 & 1 & 1 & 1 \\
\hline$\stackrel{\stackrel{P}{F}}{F}$ & 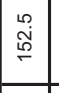 & \begin{tabular}{|l} 
\\
$\stackrel{N}{N}$ \\
\end{tabular} & $\begin{array}{l}0 \\
\text { fi } \\
\end{array}$ & $\begin{array}{l}0 \\
\stackrel{0}{0} \\
\stackrel{\rho}{\sim} \\
\end{array}$ & $\begin{array}{l}0 \\
\text { Oें } \\
0 \\
0\end{array}$ & 1 & 1 & 1 & 1 & 1 & 1 & 1 & 1 & 1 & 1 & 1 & 1 & 1 & 1 & 1 & 1 & 1 & 1 & 1 \\
\hline$\stackrel{\circ}{\circ}$ & $\mid \begin{array}{c}0 \\
\substack{0 \\
\sim \\
i}\end{array}$ & 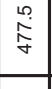 & 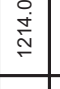 & 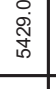 & 1 & 1 & 1 & 1 & 1 & 1 & 1 & 1 & 1 & 1 & 1 & 1 & 1 & 1 & 1 & 1 & 1 & 1 & 1 & 1 \\
\hline : & $\mid \begin{array}{l}\stackrel{0}{\dot{亠}} \\
\dot{\bar{t}}\end{array}$ & 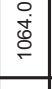 & \begin{tabular}{|c|}
0 \\
$\infty$ \\
0 \\
$\sigma$ \\
$\sigma$ \\
\end{tabular} & 1 & 1 & 1 & 1 & 1 & 1 & 1 & 1 & 1 & 1 & 1 & 1 & 1 & 1 & 1 & 1 & 1 & 1 & 1 & 1 & 1 \\
\hline$\stackrel{\circ}{\infty}$ & \begin{tabular}{|c|}
0 \\
0 \\
$\vdots$ \\
$\vdots$
\end{tabular} & 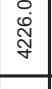 & 1 & 1 & 1 & 1 & 1 & 1 & 1 & 1 & 1 & 1 & 1 & 1 & 1 & 1 & 1 & 1 & 1 & 1 & 1 & 1 & 1 & 1 \\
\hline$\stackrel{\circ}{i}$ & \begin{tabular}{|c|}
0 \\
0 \\
0 \\
0 \\
\end{tabular} & 1 & 1 & 1 & 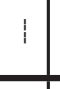 & 1 & 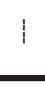 & ! & 1 & 1 & 1 & 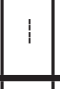 & 1 & 1 & 1 & 1 & 1 & 1 & 1 & 1 & 1 & 1 & 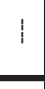 & 1 \\
\hline & $\stackrel{\circ}{\circ}$ & & 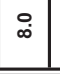 & ${ }^{\circ}$ & $\stackrel{\circ}{\circ}$ & $=$ & & & & \begin{tabular}{|l|}
$\dot{\rho}$ \\
$\stackrel{\rho}{\circ}$
\end{tabular} & $\stackrel{\stackrel{\leftrightarrow}{\circ}}{\circ}$ & $\stackrel{\stackrel{P}{-}}{\circ}$ & & $\stackrel{\circ}{\circ}$ & $\stackrel{\circ}{\dot{N}}$ & $\stackrel{\stackrel{0}{\dot{N}}}{ }$ & ì & $\stackrel{\circ}{\stackrel{\sim}{j}}$ & $\stackrel{8}{\dot{j}}$ & : & $\stackrel{i}{0}$ & $\stackrel{i}{i}$ & $\stackrel{\circ}{\stackrel{\circ}{~}}$ & i̊ \\
\hline
\end{tabular}


Appendix 3. Examples of comparison between estimated and actual strength.

\begin{tabular}{|c|c|c|c|c|c|c|c|c|c|c|}
\hline \multirow{2}{*}{$\begin{array}{r}\Phi \text { int. } \\
(\mathrm{mm})\end{array}$} & \multirow{2}{*}{$\begin{array}{l}\Phi \text { ext. } \\
(\mathrm{mm})\end{array}$} & \multirow{2}{*}{$\begin{array}{c}\text { Length } \\
(\mathrm{mm})\end{array}$} & \multirow{2}{*}{\begin{tabular}{|c|} 
Force Fv \\
$(\mathrm{N})$
\end{tabular}} & & Strength (MPa) & & \multirow{2}{*}{$\begin{array}{c}\text { Force Fh } \\
(\mathrm{N})\end{array}$} & \multirow[b]{2}{*}{ F.E.A. } & \multirow{2}{*}{$\begin{array}{c}\text { Strength (MPa) } \\
\text { Program }\end{array}$} & \multirow[b]{2}{*}{ Error \% } \\
\hline & & & & F.E.A. & Program & Error \% & & & & \\
\hline 12.50 & 13.58 & 23.0 & 4052 & 7611.0 & 7563.2 & 0.63 & 3689 & 10364.0 & 10261.0 & 0.99 \\
\hline 6.32 & 15.64 & 38.0 & 5001 & 72.7 & 74.5 & -2.48 & 4259 & 55.2 & 56.5 & -2.30 \\
\hline 16.27 & 19.65 & 50.0 & 3210 & 397.9 & 387.6 & 2.59 & 6215 & 1072.6 & 1062.1 & 0.98 \\
\hline 16.95 & 25.34 & 51.0 & 5489 & 130.8 & 133.4 & -1.99 & 4398 & 128.0 & 129.9 & -1.52 \\
\hline 7.57 & 18.45 & 36.0 & 6203 & 82.9 & 85.1 & -2.65 & 3274 & 39.2 & 39.9 & -1.68 \\
\hline 9.32 & 10.83 & 45 & 2584 & 974.3 & 994.2 & -2.04 & 3025 & 1660.7 & 1619.9 & 2.46 \\
\hline 6.22 & 8.24 & 21 & 3846 & 1275.7 & 1246.1 & 2.32 & 4244 & 1879.7 & 1956.8 & -4.10 \\
\hline 12.3 & 14.1 & 29 & 3802 & 2051 & 2085 & -1.66 & 4220 & 3264.6 & 3336.2 & -2.19 \\
\hline 7.46 & 28.78 & 43 & 6548 & 29.31 & 28.8 & 1.74 & 7802 & 23.1 & 22.5 & 2.56 \\
\hline 26.2 & 28.42 & 35 & 2450 & 1498.4 & 1489.5 & 0.59 & 3240 & 2967.7 & 2817.4 & 5.06 \\
\hline
\end{tabular}

Force $\mathbf{F v}$ is the load inducing fracture in the vertical plane.

Force $\mathbf{F h}$ is the load inducing fracture in the horizontal plane.

F.E.A. stands for the strength calculated by finite elements method.

Program stands for the strength calculated by using the free proposed program.

fracture loads. If an approximated strength value is sufficient (error less than 5\%) we propose a simple calculation method using tabulated coefficients.

\section{References}

[1] Ayal de S. Jayatilaka, Fracture of Engineering Brittle Materials, Appl. Sc. Pub. London (1979) 117-134

[2] J.C. Glandus, Meaning of the Biaxial Flexure Tests of Discs for Strength Measurements, J. Phys. Col. C1 (47) (1986) 595-600

[3] J.C. Jaeger, E.R. Hoskins, Rock Failure under the Confined Brazilian Test, J. Geophys. Res. 71 (1966) 2651-2659

[4] R.H. Marion, J. Keith Johnstone, A Parametric Study of the Diametral Compression Test for Ceramics, Am. Ceram. Bul. 56 (1977) 998-1002

[5] G. Hondros, The Evaluation of Poisson's Ratio and the Modulus of Materials of Low Tensile Resistance by the Brazilian Test with Particular Reference to Concrete, J. Appl. Sci. 10 (1959) 243-268
[6] Ö. Vardar, I. Finnie, An Analysis of the Brazilian disk fracture test using the Weibull Probabilistic treatment of brittle strength, Int. J. Fract. 11 (1975) 495-508

[7] G. Taguchi, Y. Wu, Introduction to Off-line Quality Control. Central Japan Quality Control Association, 1985, 4-10-27 Meieki Nakamura-ku Nagaya

[8] J.P. Brousse, Détermination des propriétés de rupture fragile des matériaux céramiques, Application au cas de l'alumine, CNAM Report, University of Limoges, 1981

[9] N.A. Weil, I.M. Daniel, Analysis of Fracture Probabilities in non Uniformly Stressed Brittle Materials J. Am. Ceram. Soc. 47 (1964) 268-274

[10] W.A. Nash, Theory and Problems of Strength of Materials, Schaum's outline, Mac Graw-Hill Inc., 1994

[11] B. Villechaise, Étude bidimensionnelle des contacts larges entre domaines élastiques finis, Thesis, I.N.S.A. Lyon, 1981

[12] J.C. Glandus, Rupture fragile et résistance aux chocs thermiques de céramiques à usage mécanique, Thesis, University of Limoges, 1981 